\title{
El paPEl de laS corporaciones transnacionales EN EL PROCESO DE DIFUSIÓN DE LA TECNOLOGÍA ${ }^{1}$
}

\author{
Nelson Rúa Ceballos ${ }^{2}$
}

\section{Resumen}

Este trabajo explora la problemática de la difusión de la tecnología en cuanto a las posibles vías o canales para que ella ocurra, las formas que adquiere, de acuerdo con las condiciones y potencialidades de los países en desarrollo, y sus barreras, entre otros asuntos, analizando el papel que las corporaciones transnacionales pueden jugar respecto de la difusión, como clave para la innovación, bien porque la promueven o bien porque la obstaculizan. Metodológicamente, se analizan algunas acciones que favorecen la difusión y la incorporación de las transnacionales y el impacto de la inversión extranjera directa en dicho proceso.

Por otra parte, se aborda el estudio de la naturaleza de la difusión, mediante el concepto de transferencia de tecnología, fundamentalmente, $\mathrm{y}$ se ponen de manifiesto las condiciones que facilitan que una vía de difusión pueda ser más eficiente que otra. Otra cuestión es el análisis de la incidencia que la protección de la propiedad intelectual tiene

$1 \quad$ El término "tecnología" se asume aquí en el sentido de conocimiento tecnológico (tácito y explícito) y no como producto tecnológico (en términos de tecnofactos como: red, sistema, equipo, instrumento, herramienta, dispositivo, componente, etc.) o como proceso tecnológico (conjunto de métodos o procedimientos de índole tecnológica).

2 Ingeniero Electrónico de la Universidad de Antioquia (Medellín-Colombia) con áreas de énfasis en bioingeniería y telecomunicaciones, DEA en Economía de la Innovación de la Universidad del País Vasco UPV-EHU (Donostia-España), candidato a doctor en Estudios de Ciencia y Tecnología y Gestión de la Innovación Tecnológica de la UPV-EHU (Donostia-España); Investigador, Diseñador y Desarrollador de tecnología biomédica, ex empresario del sector electrónico y electromédico, catedrático universitario, ex decano de programas de tecnología en mantenimiento de equipo biomédico, telecomunicaciones, electrónica y ciencias básicas, ex decano de la Facultad de Ciencias del ITM, ex director del Grupo de Investigación en Gestión Tecnológica -GT-(Categoría C en Colciencias), actualmente Vicerrector del Instituto Tecnológico Metropolitano ITM (Medellín-Colombia). Correo electrónico: nelsonrua@itm.edu.co.

Fecha de recepción: 14 de julio de 2008

Fecha de aceptación: 11 de agosto de 2008 
sobre la difusión, dado que ello afecta la decisión de una transnacional para establecerse en un país, y la intención misma de la inversión extranjera directa. Un resultado esperado es comprender cómo la difusión se afecta, por el proceso de transferencia de tecnología y por las barreras de la propiedad intelectual, y concluir con la adopción de elementos para el manejo de las vías de difusión, las relaciones con las corporaciones transnacionales y la regulación en materia de protección a la propiedad intelectual.

\section{Palabras clave}

Difusión de tecnología, Corporación transnacional, Transferencia de tecnología, Inversión extranjera directa, Derechos de propiedad intelectual.

\section{Alostract}

This paper explores several problems about the diffusion of the technology as well as the possible routes or channels to make it happens, the forms which it acquires, based on the conditions and potentialities of the developing countries, and their barriers, among others, analyzing the role that the transnational corporations can play regarding to the diffusion, as a key factor for the innovation, either because they promote it or because they prevent it. It is analyzed methodologically some actions that favour the diffusion and the incorporation of the transnational corporations and the impact of the direct foreign investment in this process.

On the other hand, the study of the nature of the diffusion is approached, by means of the technology transference concept and it is stated the best way that facilitate its diffusion. Another issue is the analysis of the incidence that the protection of the intellectual property has on the diffusion since it affects the decision of a transnational company to settle down in a country, and the desire of the direct foreign investment. An expected result is to understand how the diffusion is affected, by the process of technology transference and by the barriers of the intellectual property, and to conclude with the adoption of elements for the handling of the diffusion routes, the relations with the transnational corporations and the regulation of the intellectual property.

\section{Key words}

Diffusion of technology, Transnational corporation, Transference of technology, Direct foreign investment, Intellectual property rights. 


\section{INTRODUCCIÓN}

En relación con el estudio de la difusión de la tecnología, dentro de un marco de globalización, se debe analizar el importante papel que la inversión extranjera directa (IED) y las corporaciones transnacionales (CNT) del campo tecnológico pueden jugar respecto de aquella, como elemento crucial para la innovación y la economía de un país. En este contexto es importante aún analizar bajo cuáles condiciones una vía de difusión puede ser más eficiente que otra de acuerdo con las situaciones particulares y posibilidades de una región o país y con la naturaleza y políticas de las transnacionales o de los inversionistas interesados en ingresar a los mercados existentes allí.

Otra cuestión central tiene que ver con el polémico tema de la regulación de la protección a los derechos de propiedad intelectual, por ser éste un elemento crucial que incide sobre la decisión de una transnacional para establecerse en un determinado país, e incluso para aquellos que pretenden hacer inversiones directas en el extranjero para promover proyectos de desarrollo tecnológico. Esto exigiría analizar la incidencia que dicha protección de derechos tiene sobre la difusión, y establecer más claramente si hay una relación directamente proporcional entre un política rigurosa de protección a los derechos de propiedad intelectual y el establecimiento de una corporación transnacional o de la inversión extranjera directa en un país y de si ello mejora o desmejora su capacidad innovadora; es decir, si una regulación fuerte en materia de protección a los derechos de propiedad intelectual realmente incrementa la transferencia de tecnología a nivel internacional (Branstetter et al., 2006).

Otro problema es determinar cuál es el papel de las transnacionales en el proceso de innovación y si es significativo su aporte para una nación en desarrollo o si, por el contrario, dichas corporaciones contribuyen a acrecentar aún más la 'brecha tecnológica' y fortalecer los lazos de tecnodependencia y tecnosumisión (Ancarani, 1995) que aquejan a las naciones menos desarrolladas. 
Es menester resaltar, dado lo anterior, que son muchas las cuestiones por resolver en el marco de los problemas planteados, algunas de las cuales se responden en este trabajo y otras requerirán adelantar otras investigaciones para dar respuesta a preguntas como las siguientes: ¿Cuál es el rol de las corporaciones transnacionales dentro de la globalización? ¿Cómo inciden las transnacionales en la difusión del conocimiento y cuál es el impacto como actores activos de dicha difusión para la innovación en un país en desarrollo? ¿Cuáles son las vías de difusión del conocimiento más adecuadas para una región o país de acuerdo con sus condiciones particulares de desarrollo? ¿De qué manera las leyes de protección a la propiedad intelectual favorecen el ingreso de las transnacionales y la inversión extranjera directa en una determinada región o país?

\section{Concepto de difusión ${ }^{3}$ y otros conceptos conexos}

La tecnología es vista como el ingrediente central en el desarrollo económico pero no el único y, mientras tanto, se está dando una tendencia creciente en el sentido de que la tecnología y las decisiones que de ella se generan, tomadas en las empresas de negocios globales, particularmente en las corporaciones transnacionales, afectan las economías de la localidades y regiones así como las naciones (Maleck, 1991) y en los grupos financieros interesados en incursionar en mercados exteriores a través de la Inversión Extranjera Directa (IED) ${ }^{4}$.

3 La difusión emerge aquí como una de las vías centrales y necesarias, mas no es la única, para cumplir con los requerimientos de universalización que la información y el conocimiento deben satisfacer si pretendemos hablar de globalización del conocimiento o, por lo menos, del tránsito hacia un nuevo modelo de economía y de sociedad. En este trabajo nos centramos en el papel de las transnacionales frente a dicha vía y la barrera constituida por los derechos de propiedad intelectual.

4 La Inversión Extranjera Directa (IED), es un concepto definido por el FMI en 1993 del siguiente modo: "La inversión directa se refiere a la inversión que se hace para adquirir un interés perdurable en una empresa que opera en una economía que no es la del inversionista, y el propósito de este último es tener una voz efectiva en la administración de la empresa". Este concepto expresado de este modo, es muy 
El concepto simple de difusión, en materia de conocimiento tecnológico e innovación, podemos encontrarlo, de manera pragmática, en el manual de Oslo (OECD, 2005). El manual, en su tercera versión, excluye los cambios de menor importancia o con insuficiente grado de novedad. Sin embargo, una innovación no necesita ser desarrollada por la propia empresa, sino que se puede adquirir a otras a través del proceso de difusión, un procedimiento por el que las innovaciones se extienden, desde su primera práctica, a través del mercado o de otros canales o vías, a los diversos consumidores, países, regiones, sectores, mercados y empresas que constituyen su objetivo. Sin la difusión, una innovación no tiene ningún impacto económico. El manual excluye como novedad la difusión de una nueva tecnología entre distintas áreas o fábricas de una misma empresa después de su adopción inicial o comercialización en una de ellas.

Conceiçao (2003) sostiene, por su parte, que la difusión es el proceso mediante el cual las innovaciones son traducidas en impacto económico amplio, en la medida que más y más personas y empresas consuman y usen los nuevos productos o procesos resultantes de la innovación tecnológica. Visto así, parece claro que la tecnología impacta el funcionamiento de la empresa, el crecimiento, el bienestar, etc. (Karshenw, 1995). Por ello, la relación entre el funcionamiento de la empresa y el proceso de difusión de conocimiento requiere mayor investigación y evidencia empírica (Karshenw and Stoneman, 1995).

En la literatura hay abundantes trabajos que dan cuenta del análisis de la relación invención $\rightarrow$ innovación $\rightarrow$ cambio técnico (Ruttaro, 2001) en donde la difusión y la transferencia juegan un papel esencial. El cambio técnico conduce a niveles de desarrollo, sin duda, mayores que los existentes antes de tal suceso, o conjunto de sucesos. Las fuentes de cambio técnico pueden ser de tres tipos

limitado porque no toma en cuenta las variables que inciden en la toma de decisiones sobre inversión y, sobre todo, otras motivaciones distintas a las de tener una voz efectiva, así como las consideraciones tecnológicas que subyacen en un proceso de tan compleja naturaleza. 
(Ruttaro, 2001): el proceso de invención e innovación, la innovación institucional y técnica y la adopción, difusión y transferencia de tecnología. Esta última es especialmente importante en relación con el rol de las corporaciones transnacionales de tecnología en el proceso de difusión.

En la segunda no nos detendremos y digamos que la primera, la generación de nuevas tecnologías, tiene que ver más con las fuerzas que determinan la tasa a la cual las nuevas tecnologías son creadas y la forma que ellas adquieren (Jeffrey, 1995). La difusión, en contraste, se relaciona con la propagación de las nuevas tecnologías así creadas. La adopción de la tecnología se refiere, así mismo, a la creación de las condiciones para incorporar la tecnología al proceso de producción o a la vida cotidiana, según sea el caso. Adaptación de tecnología, para complementar lo anterior, es acondicionar la tecnología a unas condiciones o restricciones particulares, que no podrían hacerse con la tecnología original generada para su incorporación a los procesos productivos. Transferencia de tecnología real implica, por consiguiente, el "traspaso" del conocimiento tecnológico incorporado en dicha tecnología, usualmente conocimiento explícito, con propósitos de que el receptor de la misma pueda ejercer acciones de control de dicha tecnología (Jeffrey, 1995).

\section{Vías de difusión}

Son múltiples, y de muy variada naturaleza, las vías, trayectorias, canales o mecanismos, para hacer difusión, y van desde decisiones de política económica y social hasta actividades de índole tecnológica inherentes a los procesos habituales de innovación tecnológica, algunas de ellas articuladas coherentemente dentro de un sistema y otras desarrolladas de manera aislada y desarticulada.

Un estudio en Canadá (Bérubé and Mohnen, 2007), por ejemplo, encontró que las empresas que se beneficiaron de subvenciones al I + D y de créditos por exención de impuestos al I+D introdujeron más nuevos productos que sus contrapartes que solo se beneficiaron de los incentivos directos a la I+D. También hicieron más innovaciones 
en el primer mundo y fueron más exitosos comercializando sus innovaciones.

La generación e introducción de innovaciones tecnológicas son vistas ahora como el resultado de complejas alianzas y compromisos entre grupos heterogéneos de agentes (Bengt-Ake, 2003). La convergencia de los esfuerzos de una variedad de innovadores, cada uno de los cuales tiene una específica y todavía complementaria base tecnológica, puede conducir a la generación exitosa de una nueva tecnología (Freeman, 1991; Nelson, 1993). Una tecnología se difunde cuando se aplica a una variedad de diversas condiciones de usos. Ello hace que la difusión de una tecnología sea vista, de manera simple, como la escogencia de una nueva tecnología entre muchas disponibles. La difusión es el resultado de la selección de un diseño dominante por fuera de una variedad original de diferentes opciones tecnológicas, de aquí que las empresas que sean capaces de introducir nuevas tecnologías también son capaces, por lo menos en teoría, de incrementar su crecimiento y su participación en el mercado. De hecho, las condiciones cambiantes de mercado inducen a las empresas a innovar, tanto en estrategias de mercado como en tecnologías (Antonelli, 2003).

La experiencia de los tigres del sudeste asiático sugiere, por otra parte, que las políticas de fortalecimiento de la "inversión intangible" sobre un largo período hacen que simultáneamente se fortalezca la capacidad de importar, absorber y mejorar tecnologías desde el exterior (Freeman and Hagedoorn, 1995), lo cual constituye, de cierto modo, un proceso de difusión "hacia adentro" bien planteado.

\section{Transferencia de tecnología}

Tradicionalmente, dentro del proceso de difusión, se ha visto la transferencia de tecnología ${ }^{5}$ como un medio temporal para

5 La concepción de la transferencia de tecnología por parte del neófito, frente al experto, y la multidimensionalidad que ella conlleva, así como el enfoque amplio, dada su complejidad y multidisciplinariedad, es un tema tratado magistralmente por Bozeman (2000) en su famosa revisión sobre el tema, de la cual se recomienda una juiciosa lectura. 
actualizar el nivel de tecnología de una empresa, transfiriendo el hardware y el software para un proceso de producción particular a otra compañía entre un productor y un usuario (Bengt-Ake, 2003). Conceptualizada en esta forma, la innovación es reducida a la introducción de un nuevo producto o proceso en un nuevo escenario y la transferencia de tecnología se convierte en un vehículo para la promoción de la innovación sólo hasta el punto en que habilita al receptor a aprender cómo usar un nuevo proceso o para producir un nuevo producto. La innovación, sin embargo, es mucho más que esto: involucra productores y usuarios en un proceso de cambio interactivo, continuo y no lineal, que conduce a nuevas formas de pensar acerca de y cómo hacer las cosas. 'Aprender a aprender' es un componente clave de los proyectos de transferencia de tecnología si ellos están concebidos realmente para contribuir positivamente a la innovación en el largo plazo (Mytelka y Smith, 2003) a partir de procesos reales de transferencia de tecnología.

Para entender una amplia variedad de tópicos desde el crecimiento económico hasta la organización industrial, los economistas y estudiosos de la ciencia y la tecnología deben estar familiarizados, dentro del tema de la difusión, con el clásico concepto de transferencia internacional de tecnología (Mansfield, 1984). La forma tradicional en que se ha visto el proceso de transferencia internacional de tecnología es a través del concepto de ciclo de vida del producto. De acuerdo con esto, existe una secuencia completamente definida en la relación ente tecnología y comercio en donde los EE.UU. tienden a ser pioneros en el desarrollo de nuevos productos, disfrutando durante un tiempo de un monopolio virtual. Según este modelo, después de que ocurre la innovación, el innovador sirve los mercados extranjeros mediante la exportación. En la medida en que el producto madura y el mercado exterior se desarrolla, las compañías comienzan a construir plantas en el extranjero, y las exportaciones de los EE.UU. pueden ser desplazadas por la producción de las subsidiarias en el exterior ${ }^{6}$.

6 Este es el modelo desarrollado por Vernon, pero en la actualidad no explica adecuadamente la manera cómo se produce la difusión. De hecho, la globalización está conduciendo a que en muchos productos, cada vez más, el lanzamiento sea 
La transferencia de tecnología inversa, por su parte, y de acuerdo con Mansfield (1994) es la transferencia de tecnología de las subsidiarias en el extranjero a sus matrices en EE.UU. Es una actividad que se ha venido incrementando en los últimos tiempos.

Los estudios sobre transferencia de tecnología sugieren que cuando las personas se sienten motivadas para compartir la información, la dificultad suele relacionarse básicamente con la complejidad de la información (Van Alstyne y Blukley, 2006) (Stiglitz, 2006). Dos dimensiones relevantes de esta complejidad son el nivel de codificación y hasta qué punto la información que va a ser transferida forma parte de un conjunto de componentes interdependientes.

Como parte del análisis y la discusión, y como un marco de referencia, en general será importante tener en cuenta algunas tesis sobre transferencia de tecnología (De la Huerta, 1993), para comprender mejor la esencia del concepto aunque estas tesis puedan ser harto discutibles:

- La transferencia de tecnología sigue una regla o principio de supervivencia. Aquellos que desarrollan herramientas más eficientes, armas más poderosas, se convierten en una amenaza para aquellos que no las poseen.

- La transferencia de tecnología está basada en una tendencia a emular la eficiencia y los rendimientos tecnológicos. Los grupos sociales deben saber adoptar las tecnologías de sus vecinos más avanzados.

- La transferencia de tecnología conduce a asimilación y convergencia en las tecnologías. Distinguimos el primer mundo del segundo y el tercero, sobre la base de sus diferentes niveles de desarrollo tecnológico. Paradójicamente, esta diferencia no sólo distingue mundos "desarrollados" de los "en desarrollo" o

simultáneo en el país innovador y en el resto del mundo. Algunos ejemplos de esto lo constituyen los lanzamientos de la industria discográfica, la automovilística, el software, los medicamentos, la telefonía móvil celular, entre otros. 
"subdesarrollados"; también los retrotrae juntos en el sentido de establecer un estándar común que admita solamente las diferencias en niveles. Ahora, lo "desarrollado" y lo "subdesarrollado" se hace menos y menos diferente, mientras que, al mismo tiempo, se hace más y más desigual ${ }^{7}$.

- La transferencia de tecnología está acompañada por homogenización cultural. La tendencia a transferir tecnología a las áreas relativamente menos desarrolladas del mundo está acompañada por un incremento en la homogeneización de las sociedades, debido a que la transferencia de tecnología presupone y produce una transferencia de símbolos, eso es, de patrones o estándares culturales. La tendencia a un desarrollo desigual, así observado a menudo en comparaciones entre ciudades, regiones, sectores sociales -y aún en campos de la cultura y la ciencia - es confirmada y reforzada por el progreso internacional de la tecnología.

- La transferencia de tecnología no sigue su propia regla interna o no siempre obedece a la propia progresión interna al sistema tecnológico.

- La transferencia de tecnología no determina autónomamente un sistema social. El carácter supuestamente determinante del automatismo tecnológico sobre el sistema social ni es factual ni es científico. La idea de automatismo técnico es, por el contrario, una hipótesis acerca de la naturaleza del poder.

Hay una relación evidente entre la lógica de la transferencia de tecnología y el tema de la creciente brecha económico-tecnológica entre los mundos, entre el desarrollado y el menos desarrollado y la consolidación de la creciente tecnodependencia y la consiguiente

7 Se sugiere utilizar, de preferencia, el término "países menos desarrollados" (PMD), por ser más apropiado, en lugar de "países subdesarrollados" o "países periféricos", que pueden parecer algo peyorativos. También podría utilizarse el término "países en desarrollo o en vía de desarrollo" pero no siempre es claro ni fácil saber si un país menos desarrollado realmente está inmerso en un proceso real de desarrollo social, económico, tecnológico, etc. (Rúa, 2006). 
tecnosumisión resultante. La simple compra o transferencia de tecnología no es suficiente, por sí misma, para reproducir el sistema completo y ello podría estar lejos de aportar significativamente en este proceso. La extensión planetaria de la tecnología y del ideal moderno de desarrollo tecnológico exhibe una evolución contradictoria que conduce a una dificultad inherente, la cual se refiere al hecho de que la modernización en el tercer mundo no ha sido el resultado de su propio desarrollo histórico y cultural; en lugar de él, en gran parte este desarrollo ha involucrado la imitación de modelos derivados de las naciones del área del Atlántico Norte, como ha sido formalizado en los siglos 19 y 20 . La transferencia de tecnología, tal y como ha sido emprendida hasta ahora, y el progreso en general, presuponen oposición constitutiva, inequidades, crecimiento desigual, desigualdades (Bengt-Ake, 2003) en el crecimiento, así como desequilibrio y hasta desintegración; en general, asimetrías de distinto orden.

La historia ha mostrado que la transferencia de tecnología siempre ha sido un proceso incierto y complejo (Hilaire and Verna, 2006). Tradicionalmente siempre se creyó que había una diferencia entre la transferencia y la circulación, debido que el conocimiento técnico estaba embebido en los territorios de las pasadas comunidades humanas. Recientes estudios, referenciados por Hilaire y Verna (2006) han concluido que las circulaciones técnicas involucran adaptaciones constantes y traducciones en concordancia con las necesidades y opciones de los actores en juego. Puesto que los territorios no fueron entidades abstractas sino constructos humanos, el localismo distintivo siempre interfirió con la difusión.

El flujo de conocimiento dentro y a través de los países puede tener importantes consecuencias tanto para la productividad como para la innovación. Pero el comercio, claramente, no es el único conducto de los flujos nacionales e internacionales de conocimiento (Peri, 2005). Se ha encontrado, en los estudios sobre difusión del conocimiento en la frontera de la innovación tecnológica, que hay ideas en las tecnologías de la información y las comunicaciones (computadores y electrónica) que se difunden mucho más que el conocimiento promedio. Adicionalmente, nos encontramos con que los 
líderes de la tecnología generan conocimiento que también tiende a difundirse mucho más lejos. Los flujos de conocimiento son relevantes para la actividad innovativa regional, lo que ha hecho que el stock accesible externo de la I + D tenga un efecto sobre la innovación de las regiones, en un $50 \%$ o $80 \%$ tan grande como el de su propio stock de I + D. Esto que se acaba de mencionar puede ser importante para la toma de decisiones en materia de permitir el establecimiento de una corporación transnacional en determinado territorio.

Tenemos, por otra parte, el concepto de transferencia transnacional de tecnología como una nueva herramientas para la cooperación internacional y la difusión. Fomentar la transferencia transnacional de tecnología (TTT) constituye una de las principales prioridades de la Comisión Europea ${ }^{8}$, ya que con eso se estimula la innovación, lo cual se centra en los siguientes aspectos clave:

- Nuevos métodos para aumentar la eficacia y reducir el coste de las TTT

- Globalización de las TTT

- Ampliación de los servicios TTT más allá de las PYME9

- Marketing y concienciación

- El papel del capital humano

- Potencial para crear agrupaciones

- Innovación regional

- Fomento de la propia sostenibilidad de los agentes de TTT

8 La Comisión Europea (Comisión de las Comunidades Europeas) es la rama ejecutiva de la Unión Europea. Este es un órgano responsable de proponer la legislación, la aplicación de las decisiones, la defensa de los tratados de la Unión y, en general, se encarga del día a día en todo el territorio de la Unión.

La Comisión actúa bajo el esquema de un gabinete del gobierno, con 27 comisarios. Hay un Comisario por cada Estado miembro. No obstante, los comisarios están obligados a representar los intereses de la UE en su conjunto en lugar de su Estado de origen.

9 Se pretende trascender las PYME (Pequeñas y Medianas Empresas) para enfatizar la prestación de servicios con preferencia en las empresas de base tecnológica y en especial en aquellas de alto valor agregado diferenciado gracias a un alto nivel innovativo. 


\section{Rol de las corporaciones transnacionales en la difusión y su impacto sobre la innovación}

Los desarrollos administrativos y tecnológicos de los últimos quince (15) años, junto con la desregulación y la liberalización, han cambiado significativamente el balance entre la propiedad y las ventajas de internacionalización de grandes empresas y las ventajas locativas de los países (Chesnais, 1995). Se entiende por ventajas de internacionalización a aquellas que se refieren a la capacidad para hacerse a fuentes globales y contienen influencia del poder monopólico.

Como lo plantea Rouse (2004), será más conveniente utilizar el término "transnacional" en preferencia al "binacional" o "internacional", parcialmente, para evocar tan directamente como sea posible, la asociación entre formas migrantes de organización y corporaciones transnacionales. ('Transnacional' está reemplazando gradualmente el más popular adjetivo de "multinacional”, al menos en el discurso académico ${ }^{10}$ ).

Además de las finanzas globales, tal vez la imagen más común de la globalización económica sea la de la corporación transnacional (CTN): los enormes imperios corporativos que abarcan todo el planeta, con transacciones anuales que igual el total del PIB de muchas naciones (Held et al., 1999) ${ }^{11}$.

10 Es importante resaltar, sin embargo, que en la gerencia estratégica se hace una diferencia entre transnacional y multinacional. La transnacional, en este contexto, se caracteriza por tener un elevado nivel de centralización de sus actividades y también un alto grado de coordinación mientras que la multinacional permite a sus filiales mucha más autonomía, comportándose como empresas locales en los países en donde están instaladas. Michael Porter ha hecho buenos análisis en torno a este tema.

11 Actualmente las empresas no son empresas muy grandes, en el sentido de las grandes corporaciones que hemos conocido tradicionalmente. Cuando se habla de multinacional se piensa en una empresa gigante pero, en las últimas décadas, los cambios importantes que han sucedido en la economía mundial han permitido que exista una enorme cantidad de empresas multinacionales que, en realidad, son pymes. En razón de esto, se las denomina "multinacionales de bolsillo". En España, por ejemplo, se han creado muchísimas en las últimas décadas. Por otra parte, la característica de multinacional también está determinada por la dimensión del país de la empresa matriz. Así, y en contra de lo que pueda parecer a simple vista, no 
No hay duda de que las operaciones de las CTN son esenciales para los procesos de la globalización económica, concibiendo, en su sentido más amplio (complementando el concepto que se planteó más atrás), una CTN como una compañía que produce bienes o que vende sus servicios en más de un país. En su sentido más limitado, se refiere a una empresa que, por medio de una inversión extranjera directa (IED), controla y administra subsidiarias en varios países fuera de su base doméstica (Held et al., 1999).

Las revoluciones de la información y del transporte, junto con las innovaciones en las estrategias administrativas, han transformado, por su parte, la capacidad de las empresas para organizar la producción en una escala transnacional. Las CTN han estado a la vanguardia de las corporaciones que explotan las nuevas infraestructuras globales con el fin de organizar la producción internacional dentro de la empresa misma. Estos cambios han contribuido a la aparición de un "sistema de producción global, en el cual la capacidad de producción se dispersa hacia un número sin precedente de países en vías de desarrollo, así como industrializados.

El comercio intra-empresas es el comercio internacional de bienes o servicios dentro de una empresa única. Puesto que la empresa en el mercado ${ }^{12}$ mundial en este caso es, por definición, una corporación transnacional (TNC), buena parte del comercio intra-empresa en un comercio, en general, es un fenómeno relativamente reciente. Es más, es olvidado, tanto por los economistas internacionales nuevos como antiguos, debido a que ni siquiera han tenido una teoría de la corporación transnacional. Mientras que las corporaciones transnacionales ha existido durante más de 300 años, su prominencia ha emergido constantemente en los

es Estados Unidos el país con más multinacionales. Países como los escandinavos, Holanda, Bélgica o Suiza aparecen con muchas más. Otro asunto, sin embargo, es que las de los Estados Unidos sean las más grandes.

12 Frederic Jameson escribe que el término "el mercado" siempre ha tenido un significado dual, siendo en un sentido, y al mismo tiempo, una ideología y un conjunto de problemas institucionales prácticos (Milberg, 2001). 
pasados 30 años. En promedio, las corporaciones transnacionales tienen hoy mucho más subsidiarias y filiales foráneas que hace 30 años. Más importante aún, la organización de la corporación transnacional ha cambiado de manera completamente drástica durante este período. Hoy, además, la corporación transnacional ha ampliado su cobertura geográfica y ha profundizado la naturaleza de los vínculos entre filiales.

La existencia de la CTN siempre ha sido problemática, de algún modo, para la economía neoclásica debido a que se concibe simplemente como una alternativa al mercado, lo cual se presupone es el modo más eficiente de transacciones. Las CTN crean y destruyen mercados. "Las fuerzas de mercado" o "las fuerzas de la competencia internacional" pueden suscitar, por su parte, debates populares sobre política social y globalización. Las nuevas tecnologías en la comunicación, el diseño y la comunicación y administración de datos, han hecho posible la integración internacional de la corporación a niveles más profundos de su funcionamiento. Como resultado, la dinámica del negocio internacional (inter e intra CTN) puede verse como más importante que las "fuerzas de mercado" en la determinación de la división internacional del trabajo, el ingreso y el bienestar.

La relevancia, naturaleza y efectos económicos de las actividades de innovación de las corporaciones transnacionales (CTN), son tópicos altamente debatidos en la literatura actual sobre "la globalización de la tecnología" (Balcet and Evangelista, 2005). Un tema controversial tiene que ver con las estrategias de innovación de las filiales en el extranjero y el papel que juegan en los países anfitriones. En el caso de Italia, por ejemplo, hay evidencia empírica que muestra que las filiales en el extranjero y las firmas domésticas difieren unas de otras más en términos del tipo de estrategias de innovación perseguidas que en términos del rendimiento de la innovación. No obstante, las estrategias de innovación de las filiales extranjeras reflejan un alto grado de heterogeneidad, siendo afectadas por la características tecnológicas de una industria, el comercio tecnológico específico de las empresas como también por 
algunas características peculiares de la innovación italiana y los sistemas de producción, particularmente, a saber: su especialización en productos tradicionales e ingeniería mecánica combinados con su debilidad en industrias basadas en ciencia. En Italia, como ya hemos mencionado, fueron identificados tres modelos distintos de filiales extranjeras y rotuladas respectivamente como de baja tecnología, adaptativa y buscadoras de comercio (Balcet and Evangelista, 2005). Entre los tres, el adaptativo es el dominante.

De acuerdo con Archibugi and Michie (1997), y en relación con la trasferencia de tecnología y la actividad de las CTN, hay tres principales dimensiones de la tecnología global que es necesario tener en cuenta por aquellas:

- la explotación y transferencia de la innovación en los mercados internacionales

- la localización internacional de la investigación y el desarrollo (I+ D) y las actividades innovativas por las CTN

- las redes y la cooperación tecnológica internacional.

La propagación internacional y la descentralización de las actividades innovativas es afectada, en consecuencia, por los siguientes factores críticos:

- la necesidad de adaptar productos a las condiciones locales, regulaciones y restricciones locales

- buena infraestructura científica y capital humano en el país anfitrión

- el tamaño y las tasas de crecimiento de los mercados foráneos

- las estrategias de localización internacional de las actividades de I + D e Innovación por los competidores directos

- la capacidad de las CTN de manejar eficientemente redes de innovación y sistemas de investigación complejos

- adquisiciones de firmas allende las fronteras con capacidades similares o complementarias y

- el alto costo de la investigación y la ausencia de infraestructura científica en el país de origen. 
La mayor "innovatividad" de las filiales extranjeras depende, por tanto, de un efecto de doble composición: su concentración en industrias basadas en ciencia e intensivas en escala y su mayor tamaño. Puede constatarse que el modelo adaptativo es el dominante. Se sugiere que se ha dado una especie de convergencia entre las ventajas competitivas de la economía anfitriona y su 'atractividad' basada en la innovación, para las CTN. En este sentido es crucial la relación simbiótica entre las dimensiones local y global de la tecnología (Cantweell and Iamamarino, 2003), pero profundizar en estos aspectos desborda las posibilidades de este trabajo.

Ahora bien, ¿qué importancia tiene el conocimiento como factor de competitividad en las corporaciones transnacionales? es factor clave, sin duda, el papel del conocimiento en las CTN y su relación con la innovación y la competitividad, en lo cual es básico el proceso de administración del conocimiento ${ }^{13}$, tanto interno como externo en dichas empresas. Para ello se ha sugerido abordar un modelo de administración del conocimiento en tres etapas (Almeida et al., 2004):

- Los directores deben identificar las fuentes esenciales de conocimiento (entorno interno y los flujos de conocimiento en el país receptor). Esto exige el control de la competencia.

- Acceder a este conocimiento a través de la creación de conexiones o vías dirigidas a las fuentes importantes de ideas (conocimiento interno, conocimiento externo, alianzas, movilidad). Esto exige la creación y gestión de conexiones y establecimiento de redes.

- Aprovechar los mecanismos que los directores pueden utilizar para crear innovaciones, a través de la innovación. Esto exige capacidad de integración.

Las ventajas de la gestión del conocimiento en la corporación multinacional se fundamenta en la capacidad de utilizar las normas

13 El término administración del conocimiento también se encuentra en la literatura como gestión del conocimiento o gerencia del conocimiento. Los tres términos están íntimamente relacionado, y aparentemente parecieran tener la misma acepción, pero cada uno tiene características particulares que los hacen sustancialmente diferentes. 
o estándares para normalizar los procesos y formatos, la capacidad de utilizar las directivas para coordinar las diferentes unidades y la capacidad de utilizar las relaciones interpersonales entre empleados y una cultura común para facilitar la comunicación y la cooperación (Almeida and Phene, 2004). El conocimiento utilizado en la innovación por las subsidiarias extranjeras, como en el caso de las regiones de EE.UU. es predominantemente local (a nivel regional y nacional) (Almeida, 1996). De hecho, las firmas extranjeras utilizan conocimiento regional significativamente mayor que las firmas domésticas similares. Las firmas extranjeras también contribuirían, según esto, al progreso tecnológico local.

Un estudio de Almeida (1996), por otra parte, confirma la creencia ampliamente extendida de que las empresas norteamericanas utilizan plantas con base en los EE.UU. como fuente de tecnología. Los beneficios de la innovación mediante empresas extranjeras son también aprovechados hasta un nivel significativo por otras empresas extranjeras y domésticas en la misma región. De acuerdo con esto, las empresas extranjeras no pueden ser vistas primariamente como absorbedoras de conocimiento tecnológico, sino más bien, junto con las empresas domésticas, como jugadores o agentes en el continuo proceso de intercambio de conocimiento interempresarial y el desarrollo tecnológico regional. Otras investigaciones han mostrado que las empresas multinacionales tradicionalmente han confiado en el entorno interno y en el país receptor en lo referente al conocimiento necesario para la innovación. Mientras que los países receptores, en un principio, se consideraron simplemente mercados de fuentes de mano de obra barata para la multinacional, cada vez más las regiones de los países receptores se consideran fuentes potenciales de creación de nuevo conocimiento.

\section{Impacto potencial de la Inversión Extranjera Directa en el proceso de difusión de la tecnología}

Dentro del entendimiento del impacto de la Inversión Extanjera Directa (IED) en una economía hospedante o anfitriona se destaca el papel de la competencia (Enderewick, 2005). La presión del nivel 
de competencia es la clave determinante tanto de la magnitud como de la distribución de los beneficios de la IED. En consecuencia, y de acuerdo con esto, son esenciales las políticas relacionadas con la competencia; luego, buscar un nivel de "deseabilidad", la cual no puede ser evaluada independientemente del clima de inversión. En lo fundamental, hay una brecha significativa entre nuestro entendimiento de cómo pensamos maximizar la deseabilidad de la inversión entrante y lo que sucede en la práctica. Como lo sugirió la OCDE, hay una necesidad de enfatizar los beneficios del desarrollo de la IED.

Los primeros estudios de casos de la inversión directa extranjera identificaron varios canales a través de los cuales se producía la difusión desde las subsidiarias extranjeras hacia las empresas locales del país receptor de la inversión. Otras investigaciones sugieren que la importancia de los efectos de la difusión varía sustancialmente en función del país y del entorno sectorial en el que deberían producirse. Las magnitudes de los efectos de difusión que se presentan, de vez en cuando, en los estudios estadísticos nos conducen hacia un problema más importante y fundamental. Una de las funciones primordiales de los efectos de difusión es reducir la desigualdad en el fondo de conocimiento poseído por la organización emisora y receptora o, dicho de otro modo, reducir las asimetrías entre el emisor y el receptor.

Un problema importante, para la inversión extranjera y en muchas actividades es "saber qué es lo que uno no sabe". Las decisiones importantes de inversión (importar una tecnología patentada, entrar en un mercado de exportación) exigen algún fondo de conocimiento que es identificable, aunque sea probablemente costoso y, desde un punto de vista geográfico, distante.

La escasez de conocimiento funciona de acuerdo con la propiedad clásica de los bienes públicos de la información y su apropiación mediante ingeniería inversa, inventando o introduciendo innovaciones alrededor de las tecnologías patentadas, y mediante otras acciones similares. La investigación realizada para el caso de los países industriales sugiere una considerable filtración, 
desde las subsidiarias extranjeras, de dichos activos vinculados al conocimiento (Mansfield y Romeo, 1980).

Es muy probable que estos resultados no sean representativos respecto de lo que pasa normalmente en las empresas locales de los PMD. En aquellos lugares donde, en general, el conocimiento relevante está muy lejos de la frontera global, las empresas locales no padecen la falta de fuentes de información que pueda haber (cada una con su correspondiente precio). La filtración procedente de las subsidiarias extranjeras que más les podría ayudar sería la oportunidad que tienen de ver lo que es factible: Que un producto de mejor calidad puede ser fabricado y vendido a los precios vigentes, que los bienes producidos localmente se pueden exportar con beneficios a determinados mercados extranjeros, que un producto diseñado en el extranjero se vende bien en el mercado local (incluso antes de adaptarlo) son datos importantes para la empresa local que está considerando la posibilidad de hacerse con información costosa y que, por otra parte, no tiene otra forma de conocer su probable valor.

Un estudio de Kinishita y Hui (2006) analizó los efectos de la inversión extranjera directa (IED) sobre el crecimiento económico cuando las suficientes provisiones de infraestructura se convierten en un pre-requisto. Se mostró que los 'spill-overs' de tecnología ${ }^{14}$, vía IED, toman lugar sólo cuando el país anfitrión tiene el suficiente nivel de infraestructura. De lo contrario, el efecto de la IED sobre el crecimiento no es notorio. Se corrobora que la IED es un importante canal de difusión de la tecnología internacional una vez que se toma en cuenta la capacidad absortiva del país hospedante o anfitrión. Hay

14 Se asume aquí los spillovers tecnológicos o "spill-overs" de tecnología como el conjunto de externalidades positivas generadas por las empresas cuando realizan inversión en investigación y desarrollo (I+D). Este proceso es entendido, por consiguiente, como el desbordamiento de la I $+\mathrm{D}$ en el mismo sector o diferentes sectores, y que se verá reflejado de manera indirecta en las utilidades operacionales de las otras empresas en el mercado.

La fuente de los spillovers es la posibilidad de difusión del conocimiento entre las empresas. Estas externalidades toman lugar a través de la movilidad de empleados entre empresas, espionaje industrial o ingeniería inversa (cuando se toma un producto y se descompone para aprender cómo fue construido) (Tirole, 1988). 
tres factores que influyen sobre el grado de spill-overs de tecnología: atraso relativo (brecha tecnológica inicial), intensidad de la IED, y capacidad absorbente. Es importante resaltar que, en modo alguno, la IED per se sea es una panacea para el desarrollo económico sino que es el país hospedante quien debe encargarse de hacer inversiones previas en infraestructura para atraer la IED para maximizar la incidencia sobre los spill-overs de tecnología de la IED.

\section{Derechos de propiedad intelectual como barrera frente a la difusión de la tecnología}

En materia de protección a los derechos de propiedad intelectual los TRIPS -the World Trade Organisation's Agreement on TradeRelated Aspects of Intellectual Property Rights- constituyeron el más importante acuerdo sobre propiedad intelectual del siglo 20 (The Corner House, 2004). Dicho acuerdo marcó el comienzo de una completa revolución en el sentido de que los derechos de propiedad en la información fueron definidos y mejorados en la emergente economía de conocimiento con tendencia global. Los TRIPS efectivamente globalizan el conjunto de principios de propiedad intelectual ya que la mayoría de los países pertenecen a la OMC, que los administra, lo que además ha conducido a que los derechos de propiedad intelectual emerjan como un indicador de importancia relativa para medir el grado de desarrollo en los países menos desarrollados ${ }^{15}$. Hoy tenemos brechas: la digital, la del acceso a los medicamentos, la idiomática, la tecnológica y la de entre aquellos que evitan los impuestos trasladando sus derechos de propiedad alrededor del mundo y aquellos que simplemente tienen que pagar por ellos. Los derechos de propiedad intelectual, y los TRIPS especialmente, van más allá de la justa recompensa para un inventor individual. Incluyen, además, amplias desigualdades en los ingresos, excesivos beneficios, el poder y la influencia de los grandes negocios sobre el gobierno, la pérdida de la soberanía nacional, la globalización, temas morales acerca del uso de la

15 Así ha sido contemplado en un estudio realizado por Lall (2003). 
biotecnología, la seguridad alimentaria, la biodiversidad, el desarrollo sostenible, la autodeterminación de los pueblos indígenas, el acceso a los servicios de salud y los derechos de los ciudadanos a los productos culturales, entre muchos otros.

Por otra parte, el declive de la respetabilidad moral de los derechos de propiedad intelectual ha sido acompañado por el incremento de los niveles de activismo transnacional contra el uso y extensión de los regímenes de propiedad intelectual, casi hasta un nivel "feudal" de pago de tributos. Si las desigualdades del "feudalismo de la información" son resistidas exitosamente, podrán formarse alianzas o coalisiones globales. Los TRIPS solo fueron posibles debido a una élite de compañías basadas en el conocimiento, o intensivas en tecnología de EE.UU, Europa y Japón (los "tres grandes") que establecieron sus diferencias y marcharon unidas en su necesidad de una protección global de la propiedad intelectual. Resistir este paradigma requiere que diversos grupos y comunidades se unan en una política global que "obligue" a los gobiernos a diseñar regulaciones de derechos de propiedad intelectual que sirvan al bienestar y a las libertades básicas de los ciudadanos y no como instrumentos de consolidación de las corporaciones transnacionales en detrimento de la innovación y el progreso de los países menos desarrollados mediante el fortalecimiento de lazos de tecnodependencia y tecnosumisión cada vez más onerosos.

Es así como la definición clara de los derechos de propiedad intelectual constituye un elemento clave para el desarrollo de la economía de mercado (De los Reyes, 2002). Al fin y al cabo, una transacción económica no es más que un intercambio en el mercado de dos paquetes de derechos de propiedad y es el valor de esos derechos de propiedad, en última instancia, el que determina el valor de la transacción. En la especificación de estos derechos radica, por lo tanto, la base del funcionamiento correcto de la economía de mercado, motor de la doctrina económica neoliberal imperante hoy en el mundo. Por otra parte, el desarrollo económico, a la vez que ha aumentado la cantidad de bienes y servicios de que 
se dispone en las sociedades avanzadas, ha variado la naturaleza de los mismos, de un modo y manera tales que hoy día los productos no materiales o intangibles, es decir los basados en el conocimiento, han alcanzado tal importancia que definimos a las sociedades avanzadas como sociedades de la información o economías del conocimiento, si bien esta denominación es apenas una pretensión y surge, a continuación, el debate de si estamos en la transición hacia aquellas sociedades o si ya estamos en ellas.

Los derechos de propiedad intelectual, en lo fundamental, confieren al propietario del producto en cuestión el derecho exclusivo a utilizarlo. Crea un monopolio. El propietario de esos derechos puede, por descontado permitir que otros lo utilicen, normalmente, previo pago de una determinada suma de dinero. (Stiglitz, 2006) La regulación de la propiedad intelectual se ha ideado, en esencia, para garantizar que inventores, escritores y otra personas o empresas que invierten su dinero y su tiempo en actividades creativas reciban algo a cambio de su inversión.

Tal y como están concebidos en la actualidad, los derechos de propiedad intelectual crean un monopolio (Stiglitz, 2002, 2006). El monopolio genera rentas monopolistas (rentas excesivas) y son esas rentas las que, presuntamente, suponen un incentivo para iniciar o proseguir las investigaciones. Con el aprovechamiento del saber de qué hacen uso los monopolios se asocian ineficiencias particularmente graves, porque el saber, o el conocimiento, es lo que los enemigos de dichos monopolios llaman un "bien público" porque, potencialmente, todos podemos beneficiarnos de él y su uso no debería conllevar ningún costo. Por eficiencia económica, el saber debería estar más disponible, mientras que la legislación nacional y la regulación internacional de la propiedad intelectual tienden a restringir el acceso a él.

Cuanto mayor sea el alcance de la propiedad intelectual (cuantas más cosas puedan patentarse, cuanto más abarquen las patentes), mayor la recompensa, el premio, de quien posee la patente, -y mayor el alcance del monopolio y de los costos que comporta- (Stiglitz, 2006) de lo cual se deduce que la monopolización 
no sólo puede provocar un estancamiento de la ineficiencia, sino reducir la innovación, contrario a lo que plantean los defensores de una legislación fuerte en materia de protección a los derechos de propiedad intelectual. En los casos de pugnas en los tribunales, la propiedad intelectual se salda con pérdidas generalizadas; la economía pierde a corto plazo, puesto que los altos precios propios de la situación monopolística reducen el bienestar; y a largo plazo, en razón de que se reduce la innovación (Stiglitz, 2006).

Es importante, como marco de referencia, identificar los determinantes de la localización de las actividades o procesos de I + D de las empresas multinacionales (EMN) clásicas ${ }^{16}$ de los EE.UU. (Sanyal, 2001), en relación con la incidencia de los derechos de propiedad intelectual. Es esencial, también, determinar el impacto de la protección de los derechos de propiedad intelectual sobre la inversión en I + D por parte de las multinacionales tanto en economías desarrolladas como en aquellas en desarrollo. Un gran hallazgo en este análisis tiene que ver con que en las economías en desarrollo, las EMNs primariamente investigan sobre el alcance de la inversión extranjera directa, mientras localizan sus instalaciones de I + D en otros lejanos sitios. Por otra parte, para los países desarrollados, el alcance de las leyes de protección de patentes es el principal factor para que las EMNs decidan invertir en I + D. Otro hallazgo con el que nos encontramos es que en esas economías en desarrollo, la localización de la I + D está motivada por la necesidad de adaptar productos y procesos a las condiciones de los mercados extranjeros. Algunas implicaciones de política son determinantes para la entrada de la inversión extranjera en los países en desarrollo que estén aspirando a tener centros de Inversiones de I + D por parte de las compañías multinacionales.

16 Se plantea aquí, en este párrafo, el término clásico "Empresa Multinacional" (EMN o MNE por su sigla en inglés) dado que es todavía muy común en la literatura, pero de acuerdo con lo visto preferiremos el de CTN, por ser más apropiado y responder mejor al concepto de transnacionalización de las empresas. 
Pero Mientras Stiglitz (2006) sostiene que una protección excesiva de la propiedad intelectual puede ralentizar ${ }^{17}$ la innovación, los defensores de esa política sugieren que, por el contrario, promueve la investigación.

La propiedad intelectual no es ni debe ser un fin en sí mismo, sino un medio para un fin: en teoría, mejorar el bienestar social promoviendo la innovación (Heald, 2004). El famoso estudio empírico de Mansfield de 1994 de la American Businees Executive for the International Finance Corporation es citado profusamente por la proposición de que si los países en desarrollo elevan su nivel de protección de la propiedad intelectual (especialmente las patentes), ellos atraerán inversión extranjera y transferencia de tecnología. Heald (2005), por el contrario, lanza una mirada escéptica a dicho trabajo y concluye que el mundo en desarrollo debe ser muy receloso con el persistente clamor de que su estudio de referencia de los hacedores de decisiones corporativas soporten una implementación maximalista y mejoren la estrategia a través de todas las áreas relativas a la propiedad intelectual.

En lugar de acoger la investigación de Mansfield, una estrategia más racional para los países en desarrollo debe tener en cuenta los costos y beneficios de la protección en el contexto de su situación económica particular. Dependiendo de las categorías en las que se agrupa la propiedad intelectual sujeta al acuerdo de TRIPS, los países en desarrollo deben considerar seriamente el cumplimiento mínimo de las provisiones de patentes de los TRIPS y el cumplimiento máximo con la provisión del secretismo comercial de los TRIPS. Bajo ninguna circunstancia, un país en desarrollo debe aceptar las afirmaciones confidenciales hechas por algunos autores que adoptan una postura maximalista hacia toda protección que estimule la inversión extranjera. Un análisis de la ley-por la ley-,

17 En términos generales, en la medida en que impiden la difusión y el aprovechamiento del saber, las patentes ralentizan la investigación de continuidad, esto es, las innovaciones que se basan en otras innovaciones, y, puesto que casi todas las innovaciones surgen de innovaciones anteriores, también ralentizan el progreso tecnológico global (Stiglitz, 2006). 
orientado a favorecer el conocimiento como bien público, siempre sería necesario y conveniente.

Por el contrario, otros articulistas, en la línea de Mansfield, piensan que los países con una política fuerte en materia de protección a la propiedad intelectual tienden a experimentar un continuo flujo de nuevas empresas de alta tecnología que entran a la base industrial. Esto es así, dicen, porque la protección a la propiedad intelectual asumida por un país directamente se relaciona con la cantidad de desarrollo técnico y transferencia dentro del país en desarrollo (Sherwood, 2002). Surge aquí el importante caso de las patentes.

¿Patentar o no patentar? Esta es la cuestión. Si el sistema de patentes no es tan costoso, y cada día es más accesible y aunque complejo, sus dificultades no deberían ser mayores que muchos otros procesos burocráticos. ¿Por qué no se patenta profusamente, entonces, en determinado país? Es la pregunta que se hace Arias (2007), en España. Son principalmente las empresas de base tecnológica las que no están aumentando su participación en el sistema de patentes y surge la pregunta de si se trata de puro desconocimiento, miedo a un sistema que no se considera fiable, estrategia empresarial o tipología de empresas que operan en el país. Después de comentar estos aspectos cabe lanzar una cuestión: si el sistema de patentes no es tan costoso (supuestamente, teniendo en cuenta la relación costo-beneficio), cada día es más accesible y aunque complejo, y sus dificultades no son mayores que muchos otros procesos burocráticos ¿Por qué no se patenta en un país como España, por ejemplo?

Desde el punto de vista de las CTN, la protección de los derechos de propiedad intelectual es importante para las empresas, ya que favorece la competitividad y la innovación tecnológica (de ellas). La legislación ya incluye protección contra la piratería y la falsificación, y también existen soluciones de software disponibles. Este modelo se basa en un enfoque de propietario. No obstante, muchos usuarios son partidarios del suministro abierto, un modelo diferente destinado a compartir los resultados de la innovación 
del modo más amplio posible. Esta controversia hace mucho más difícil la búsqueda de soluciones que se adapten a las necesidades de todos. En lo que respecta a las descargas de los usuarios más jóvenes, los legisladores tendrían que encontrar un equilibrio entre la penalización y la información y la educación.

Es interesante anotar, finalmente, que la diversidad de sistemas de derechos de propiedad intelectual refleja, de algún modo, la diversidad de sistemas de innovación (Foray, 1995). Así, la política inspirada en la estandarización de los derechos de propiedad intelectual debería estar basada en la estandarización de los sistemas de innovación; o dicho de otro modo, el sistema de incentivos global y uniforme puede deteriorarse al ser acoplado a los procedimientos y condiciones de innovaciones en ciertos países. Las especificidades de los sistemas de innovación, a los cuales está enlazado cada régimen de propiedad intelectual, son un obstáculo a la estandarización de estos regímenes. Para alcanzar un régimen global, único, se requeriría una reducción en la diversidad de los sistemas de innovación mismos. No obstante, este tipo de diversidad es una característica clave del proceso de cambio científico y tecnológico y no es tema de discusión en este trabajo.

\section{A MANERA DE CONCLUSIÓN}

Una vez adquirido el concepto de difusión de la tecnología y de una de sus vías más prominentes: la transferencia de tecnología, se hace imperativo para las empresas de una nación y para los hacedores de política pública comprender el manejo de distintas vías de difusión (con el propósito de privilegiar aquellas que mejor se adapten a sus condiciones particulares y permitan un mayor rendimiento en el tiempo), la manera como se deben manejar las relaciones con las corporaciones transnacionales (desde la promoción de alianzas estratégicas para la innovación compartida hasta las formas de contratación que mejor posibiliten el proceso real de transferencia de tecnología) y la regulación en materia de protección a la propiedad intelectual (lo suficientemente equilibrada para que permita la atracción de la inversión extranjera directa y 
el asentimiento de las corporaciones transnacionales sin que ello implique deteriorar significativamente la difusión que beneficie realmente la innovación).

Igualmente se requiere analizar también la importancia del tamaño de la empresa y de las acciones gubernamentales de política pública, en materia económica y de ciencia y tecnología, para favorecer realmente la difusión y, especialmente, la incorporación de las corporaciones transnacionales (CTN) o de la inversión extranjera directa (IED) en la economía de un país y en sus procesos de innovación.

Se ha demostrado, sin embargo, que sí hay una relación directamente proporcional entre un política rigurosa de protección a los derechos de propiedad intelectual y el establecimiento de una corporación transnacional o de la inversión extranjera directa en un país y que lo primero desmejora la capacidad innovadora; es decir, una regulación fuerte en materia de protección a los derechos de propiedad intelectual realmente posibilita el asentamiento de una CTN o la incursión de la IED pero no la transferencia real de tecnología a nivel internacional (Branstetter et al., 2006), considerando aquí la tecnología como conocimiento tecnológico que implique la transferencia del control del diseño y no como el tecnofacto con información no consustancial con dicho conocimiento.

Si bien hay otras acciones de gobierno distintas a las subvenciones al $\mathrm{I}+\mathrm{D}$ y de créditos por exención de impuestos al $\mathrm{I}+\mathrm{D}$ para apoyar procesos de difusión de tecnología, estas alternativas suelen ser mejores que los incentivos directos a la I $+D$, como lo ha demostrado la experiencia canadiense, pero no necesariamente son las únicas vías de promoción y no puede esperarse que apliquen con igual éxito en otro país.

Sin duda, las mejores posibilidades para la difusión se presentam a partir de la generación e introducción de innovaciones tecnológicas como el resultado de complejas alianzas estratégicas y compromisos entre grupos heterogéneos de agentes, dado que una tecnología (como tecnofacto más no como conocimiento tecnológico) se difunde cuando se aplica a una variedad de diversas condiciones 
de uso, siendo éste un factor importante en el proceso de difusión, por lo menos desde el punto de vista de la corporación transnacional interesada en que ello ocurra, así ello no signifique lo mismo (en materia de innovación) para el hospedante o anfitrión (trátese de una empresa o de un región o país).

Como lo sugiere la experiencia del sudeste asiático las políticas de fortalecimiento de la "inversión intangible" sobre un largo período hacen que simultáneamente se fortalezca la capacidad de importar, absorber y mejorar tecnologías desde el exterior con un impacto positivo sobre la difusión en beneficio de la nación receptora.

Finalmente, es claro que una mayor restricción a la universalización del conocimiento puede ser ocasionada por la manera como las corporaciones transnacionales actúan en el proceso de difusión del conocimiento tecnológico, particularmente en el tema de la transferencia de tecnología, incrementada por el papel de los derechos de propiedad intelectual, en donde se pone en evidencia el beneficio para dichas corporaciones o para quienes hacen inversión directa desde el extranjero sin que ello implique necesariamente un beneficio real, sin considerar el aspecto económico, por lo menos, en la difusión de un conocimiento tecnológico que impacte positivamente sobre la innovación local, regional o nacional. Es decir, desde el punto de vista de la difusión, no hay un impacto real sobre el conocimiento tecnológico asociado al objeto tecnológico de una CTN que beneficie, en materia de innovación, al país en donde aquella se asiente sino una generalización de su tecnología, como tecnofacto, con un claro beneficio económico para ella, producto de su incursión y posterior posicionamiento en dicho mercado.

\section{Bibliografía}

Almeida, P. (1996). 'Knowledge Sourcing by Foreign Multinationals: Patent citation analysis in the U.S. Semiconductor Industry', Strategic Management Journal 17 (Winter Special Issue):155-165.

Almeida, P. and Phene, A. (2004). 'The influence of the MNC and host country on innovation', Strategic Management Journal. 
Almeida, P; Grant, R.; and Phen, A. (2004). 'Conocimiento y Competitividad de las Multinacionales', Estudios Empresariales (114): 6-16.

Ancarani, V. (1995). Globalizing the world. Science and technology in international Relations, in Jasanof, S.; Markle, G., Petersen, J., Handbook of Science and Technology Studies, Sage Publications.

Antonelli, C. (2003). 'Mana trajectories and networks: shifting heuristics in the economics of innovation and new technologies', in Bengt-Ake, C.; Heitor, M. and Lundvall, (eds.) (2003). Innovation, competente Building and Social in Europe. Towards a Learning Society”, Edgar Elgar Publishing Limited, Glos.

Archibugi, D. and Michie, J. (1977). Technology, globalisation and economic performance, Cambridge University Press, Cambridge.

Arias, P. E. (2007). 'Patentar o no patentar: esa es la cuestión',Servicio de Documentación de la Oficina Española de Patentes y Marcas.

Balcet, G. and Evangelista, R. (2005). 'Global Technology: innovation strategies of foreign affiliates in Italy', Transnational Corporations, 14 (2):5391.

Bengt-Ake, C.; Heitor, M. and Lundvall, (eds.) (2003). Innovation, competente Building and Social in Europe. Towards a Learning Society", Edgar Elgar Publishing Limited, Glos.

Bérubé, Ch. and Mohnen. P. (2007), 'Are Firms That Received R\&D Subsidies More Innovative?', United Nations University - Maastricht Economic and social Research and training centre on Innovation and Technology.

Bozeman, B. (2000). 'Technology Transfer and Public Policy: a Review of Research and Theory', Research Policy (29): 627-655.

Branstetter, L.; Fisman, R.; and Fritz, C. (2006), 'Do Stronger Intellectual Property Rights Increase International Technology Transfer? Empirical Evidence from U.S. Firm-Level Panel Data', The Quarterly Journal of Economics (February): 321-349.

Cantweell, J. and Iammarino, S. (2003). Multinacional Corporations and European Regional Systems of Innovation, London: Routledge.

Caves, R. (2002). 'Los efectos de difusión en las multinacionales en los países en desarrollo: los mecanismos en acción', Revista Asturiana de Economía, (23).

Chesnais, F. (1995). Some Relationships between foreign direct investment, technology, trade and competitiveness, in HAGEDOORN, John (ed.), 
Technical Change and the World Economy. Convergente and Divergente in Technology Strategies, Edward Elgar, Hants.

Conceiçao, P.; Heitor, M.-V, and Lundavll, B.-A. (2003). 'Towards a Learning Society' en Bengt-Ake, P.; Heitor, M. and Lundvall, B. (Eds.), Innovation, competente Building and Social in Europe. Towards a Learning Society", Edgar Elgar Publishing Limited, Glos, 2003.

De la Huerta, M. G. (1993). 'Globalization: homogenization with an increasing technological gap' in Mitcham, C. (ed.) (1993). Philosophy of Technology in Spanis Speaking Countries, Kluwe Academic Publisheres, Dordrecht.

De los Reyes, M. (2002). 'Principales novedades en la regulación de los derechos de autor ante las nuevas tecnologías', en Economiaz, Revista Vasca de Economía. Especial sobre La propiedad intelectual en la sociedad del conocimiento. No. 51-2002.

Enderewick, P. (2005). 'Attacting "desirable" FDI: theory and evidence'. Transnational Corporations, Vol. 14, No. 2 (august).

Foray, D. (1995). The economics of Intellectual Property Rights and Systems of Innovation: The Persistence of National Practices Versus the New Global Model of Innovation, en HAGEDOORN, John (ed.), Technical Change and the World Economy. Convergente and Divergente in Technology Strategies, Edward Elgar, Hants.

Heald, P. (2004). 'A skeptical look at Mansfield's famous 1994 survey'. Information Ecnomics and Policy 16, 57-65.

Hilaire, L. and Verna, C. (2006). 'Dissemination of Technical Knowledge in the Middle Ages an d the Early Modern Era. New Approaches and Methodological Issues', Technoloy and Culture 47.

Kinishita, Y. and Lu, Ch-H. (2006). 'On the Role of Absorptive Capacity: FDI Matters to Growth', William Davidson Institute Working Paper Number 845, August.

Lall, S. (2003). 'Indicators of the Relative Importance of IPRs in Developing Countries', Research Policy (29): 627-655.

Mansfield, E. (1984). R \& D and Innovation: Some Empirical Findings, en Griliches, Zvi (ed.), R \&D, Patents and Productiviy, The University of Chicago Press, Chicago.

Milberg, W. (2001). Decentering the market metaphor in intenational economics, in Cullenberg, J. and Ruccio, D. (eds.), Postmodernism, economics and knowledge, Routledge, London. 
Mytelka, L. and Smith, K. (2003). 'Interactions between policy learning and innovation theory' en Bengt-Ake, C; Heitor, P., and Lundvall, B. (eds.) (2003). Innovation, competente Building and Social in Europe. Towards a Learning Society”, Edgar Elgar Publishing Limited, Glos, 2003.

OECD (2005) Oslo Manual, Third Edition, European Commission.

Peri, G. (2005). 'Detrerminants of knowledge flows and their effect on innovation'. The Review of Economics and Statistics, May 2005, 87 (2): 308-322.

Rouse, R. (2004) "Mexican migration and the social space of postmodernism", in PETER, Jackson; GRANG, Philip and DWYER, Claire, Transnational Spaces, Routledge, Oxon.

Rúa, N. (2006). La globalización del conocimiento científico-tecnológico y su impacto sobre la innovación en los países menos desarrollados. Tecnológicas, Instituto Tecnológico Metropolitano -ITM-, Medellín.

Ruttaro, V. (2001), Technology, Growth and Development, Oxford University Press, NY.

Sanyal, P. ( 2001 ). 'Intellectual Property Rights, protection and location of R \& D by Multinational Enterprises', Journal of Intellectual Capital, Vol. 5 , No. 1, pp. 59-76.

Sherwood, R. M. (2002). Global Prospects for the role of intellectual property in technology transfer. 42 idea: The Journal of Law and Technology 42, 27.

Shrum, W. and Shenav, Y. (1995). 'Science and Technology in Less Developed Countries', in Jasanof, S.; Markle, G.; Petersen, J. Handbook of Science and Technology Studies, Sage Publications.

Stiglitz, J. (2006). Cómo hacer que funcione la globalización. Taurus, Madrid.

Stiglitz, Joseph. "El malestar en la globalización”, Aguilar, Altea, Taurus, Alfaguara, S.A., Bogotá, 2002.

Tirole, J. (1988). Theory of Industrial Organization. MIT Press.

Van Alstyne, M. y Bulkley, N. (2006). 'Por qué la información debería influir en la productividad" en Castells, M. (ed.). La Sociedad Red: una vision global. Alianza Editorial, Madrid. 